\title{
Magnetic field design for floating zone crystal growth
}

\author{
K. Li, W.R. Hu* \\ National Microgravity Laboratory, Institute of Mechanics, CAS, 15, Zhong Guan Cun Road, Beijing 100080, China
}

\begin{abstract}
The magnetic fields produced by electrical coils are designed for P-doped Si crystal growth in a floating full zone in microgravity environment. The fields are designed specially to reduce the flow near the free surface and then in the melt zone by adjusting the coil positions near the melt zone. The effects of the designed magnetic fields on reducing the flow velocity and the non-uniformity of the concentration distribution in the melt zone are better than those of the case of a uniform longitudinal magnetic field, obtained by numerical simulation. It is expected to improve the radial macrosegregation and reduce the convection in the crystal growth at the same time by using the designed magnetic field. (C) 2001 Elsevier Science B.V. All rights reserved.
\end{abstract}

PACS: $81.10 ; 47.65$

Keywords: A1. Convection; A1. Fluid flows; A1. Growth models; A1. Magnetic fields; A2. Floating zone technique

\section{Introduction}

The floating full zone method is a crucible-free process, where the melt zone is confined by the melt surface tension, and is used for the growth of high quality single crystals. However, the diameter of the grown crystal is limited under terrestrial conditions. The microgravity environment provides the possibility of growing large size crystals by the floating full zone method. In this case, the thermocapillary convection driven by the surface tension gradient is dominant in the melt zone, and the buoyancy-driven convection is greatly reduced. The thermocapillary convection may be unsteady,

*Corresponding author. Tel.: + 86-10-62558226; fax:+8610-62558226.

E-mail address: wrhu@mail.imech.ac.cn (W.R. Hu). and then induces the impurity striations. In order to suppress the unsteady thermocapillary convection in the melt, a steady magnetic field, in many cases a uniform longitudinal magnetic field, is applied [1-3]. The convection in the central region of the melt zone is damped and the impurity striations in the corresponding part of the grown crystal are eliminated if the longitudinal magnetic field is strong enough [3]. The longitudinal magnetic field effectively reduces the flow across the magnetic field lines, but has no direct influence on the flow along the magnetic field lines such as on a cylindrical free surface, where the thermocapillary convection is driven, no matter how strong the longitudinal magnetic field strength is there. The relatively strong convective recirculations still persist near the free surface while the weak flow occupies the central region of the melt 
zone. Such a flow structure will induce a large nonuniformity of the concentration distribution in the melt zone, especially at the solidification interface, and result in large radial macro-segregation in the grown crystal [3]. It is important to improve the flow field structure in the floating zone, and then the non-uniformity of the concentration distribution at the solidification interface by an optimal design of the applied magnetic field [4]. This goal cannot be achieved by only increasing the strength of the longitudinal magnetic field $[3,5]$.

In the previous paper [4], the numerical simulation approach is applied to discuss the magnetic field design in a floating half zone for reducing the thermocapillary convection. In the present study, the axisymmetric magnetic fields produced by electrical coils are designed for P-doped Si crystal growth by the floating full zone method in a microgravity environment. The fields are designed specially to reduce the flow near the free surface and then in the melt zone by adjusting the coil positions near the melt zone. The effects of the designed magnetic fields on convection, temperature and concentration distributions are obtained by numerical simulation. The results show that the approach of the magnetic field design can improve the non-uniformity of the concentration distribution at the solidification interface obviously while reducing the thermocapillary convection as expected.

\section{Model}

The model of the floating full zone in the present paper is simplified as shown in Fig. 1, where a cylindrical coordinate system $(r, \theta, z)$ is used and axial symmetry $\partial / \partial \theta=0$ is assumed. The melt zone is floating between the melt and solidification interfaces at $z=0$ and $z=L$, respectively. The cylindrical single crystal and the feed rod have the same radius $R_{0}$ and the free surface of the melt zone is a cylinder with the same radius $R_{0}$. The melting interface and the solidification interfaces are assumed to be flat and kept at the constant melting temperature $T_{0}$. The dependence of the interface melting temperature on the impurity

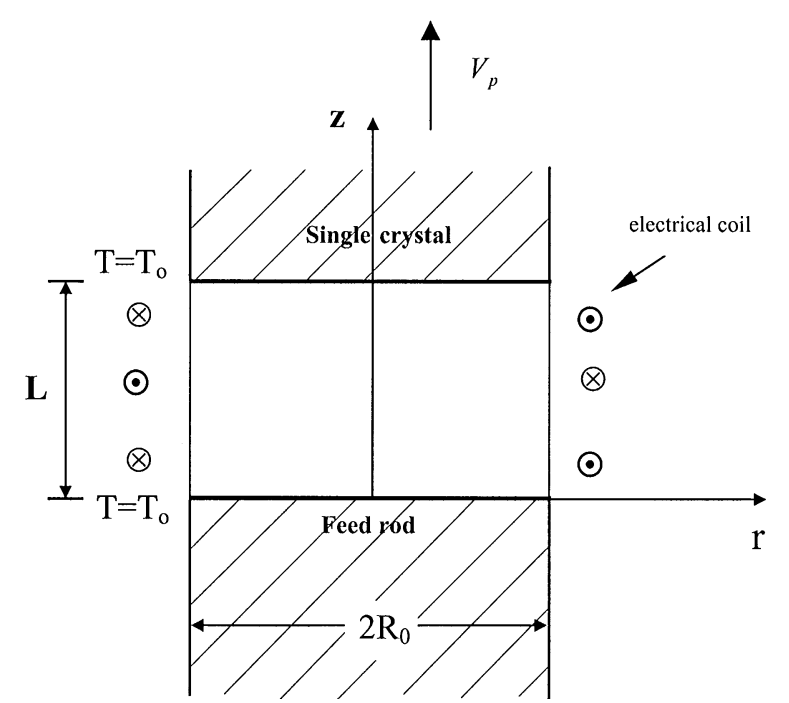

Fig. 1. Schematic diagram of a floating full zone crystal growth model.

concentration is not included, because the considered impurity concentration is dilute. The ambient temperature distribution is assumed to be Gaussian as follows:

$T_{a}(z)=T_{0}+\Delta T \exp \left\{-\left[\left(z-\frac{1}{2} L\right) / a\right]^{2}\right\}$,

where $\Delta T$ is the difference between the maximum of the ambient temperature distribution and the melting point, and $a$ is the typical width of the distribution. The feed rod is fed into the melt zone and the grown single crystal is withdrawn from the above at the same growth velocity $V_{p}$, upwards in $z$-direction.

The designed magnetic fields in the present paper are produced by several electrical coils, which are located axisymmetrically at adjusted positions near the free surface. The diameter of the coil is small and assumed to be zero for simplification. The magnetic fields produced by the electric currents of the coils are computed based on Biot-Savart's law. Because the magnetic Reynolds number is very small for semiconductors, the induced magnetic field can be neglected and the induced electric current is adopted as zero in this case. 
The non-dimensional quantities and parameters are defined as follows:

$$
\begin{aligned}
& r^{*}=\frac{r}{R_{0}}, z^{*}=\frac{z}{R_{0}}, u^{*}=\frac{u}{U_{0}}, v^{*}=\frac{v}{U_{0}}, p^{*}=\frac{p}{\rho U_{0}^{2}}, \\
& T^{*}=\frac{T-T_{0}}{\Delta T}, c^{*}=\frac{c}{c_{0}}, \vec{B}^{*}=\frac{\vec{B}}{B_{0}}, T^{*}=\frac{T_{0}}{\Delta T}, \\
& V_{p}^{*}=\frac{V_{p}}{U_{0}}, \mathrm{Re}=\frac{U_{0} R_{0}}{v}, \mathrm{Ma}=\frac{U_{0} R_{0}}{\kappa}, \mathrm{Pe}=\frac{U_{0} R_{0}}{D}, \\
& \Gamma=\frac{L}{R_{0}}, \mathrm{Bi}=\frac{h R_{0}}{k}, \mathrm{Ra}=\frac{\varepsilon \sigma R_{0} \Delta T^{3}}{k}, \\
& \text { На }=B_{0} R_{0} \sqrt{\sigma_{\mathrm{e}} / \rho v},
\end{aligned}
$$

where $(u, 0, v)$ and $\vec{B}=\left(B_{r}, 0, B_{z}\right)$ are, respectively, the vectors of velocity and magnetic field in $(r, \theta, z)$ direction; $\Gamma$ is adopted as $2.0 ; p, T, \rho$ and $c$, respectively, the pressure, temperature, density and the impurity concentration of the melt; $c_{0}$ the impurity concentration in the feed $\operatorname{rod} ; v, \kappa$ and $\sigma_{\mathrm{e}}$, respectively, the kinematic viscosity of the melt, the thermal diffusion coefficient and the electric conductivity; $h, \varepsilon, \sigma$ and $k$, respectively, the heat transfer coefficient, emissivity, Stefan-Boltzmann constant and the thermal conductivity; $D$ the diffusion coefficient. The characteristic velocity is defined as $U_{0}=\left|\sigma_{T}\right| \Delta T / \rho v, \sigma_{T}$ is the gradient of surface tension. The characteristic magnetic field $B_{0}=\mu_{r} \mu_{0} I_{0} / \pi R_{0}$, where $\mu_{r}$ is the relative magnetic permeability of the melt, and $\mu_{r}=1$ here; $\mu_{0}$ is adopted as $4 \pi \times 10^{-7} \mathrm{H} / \mathrm{m}$, and $I_{0}$ is the electric current intensity in each coil. The superscript "*" denotes the non-dimensional quantities and will be omitted hereafter for simplification. The physical properties of $\mathrm{P}$-doped $\mathrm{Si}$ are listed in the Appendix.

The thermocapillary convection in the melt zone is assumed steady and axisymmetric. The nondimensional governing equations are written as follows, and the gravity term is omitted under the microgravity condition:

$$
\frac{1}{r} \frac{\partial}{\partial r}(r u)+\frac{\partial}{\partial z}(v)=0
$$

$$
\begin{aligned}
& \frac{1}{r} \frac{\partial}{\partial r}\left(r u^{2}\right)+\frac{\partial}{\partial z}(v u)= \\
& -\frac{\partial p}{\partial r}+\frac{1}{\operatorname{Re}}\left[\frac{1}{r} \frac{\partial}{\partial r}\left(r \frac{\partial u}{\partial r}\right)+\frac{\partial^{2} u}{\partial z^{2}}-\frac{u}{r^{2}}\right] \\
& +\frac{\mathrm{Ha}^{2}}{\mathrm{Re}^{2}}\left(v B_{r} B_{z}-u B_{z}^{2}\right) \\
& \frac{1}{r} \frac{\partial}{\partial r}(r u v)+\frac{\partial}{\partial z}\left(v^{2}\right)= \\
& \quad-\frac{\partial p}{\partial z}+\frac{1}{\operatorname{Re}}\left[\frac{1}{r} \frac{\partial}{\partial r}\left(r \frac{\partial v}{\partial r}\right)+\frac{\partial^{2} v}{\partial z^{2}}\right] \\
& +\frac{\mathrm{Ha}^{2}}{\mathrm{Re}^{2}}\left(u B_{r} B_{z}-v B_{r}^{2}\right) \\
& \frac{1}{r} \frac{\partial}{\partial r}(r u T)+\frac{\partial}{\partial z}(v T)=\frac{1}{\mathrm{Ma}}\left[\frac{1}{r} \frac{\partial}{\partial r}\left(r \frac{\partial T}{\partial r}\right)+\frac{\partial^{2} T}{\partial z^{2}}\right] \\
& \frac{1}{r} \frac{\partial}{\partial r}(r u c)+\frac{\partial}{\partial z}(v c)=\frac{1}{\operatorname{Pe}}\left[\frac{1}{r} \frac{\partial}{\partial r}\left(r \frac{\partial c}{\partial r}\right)+\frac{\partial^{2} c}{\partial z^{2}}\right]
\end{aligned}
$$

The non-dimensional boundary conditions are written as

$$
\begin{aligned}
r=0, z \in(0, \Gamma): u=0, \frac{\partial v}{\partial r}=0, \frac{\partial T}{\partial r}=0, \frac{\partial c}{\partial r}=0, \\
r=1, \quad z \in(0, \Gamma): u=0, \frac{\partial v}{\partial r}=-\frac{\partial T}{\partial z}, \\
-\frac{\partial T}{\partial r}=\operatorname{Bi}\left(T-T_{a}(z)\right) \\
+\operatorname{Ra}\left[\left(T+T_{0}\right)^{4}-\left(T_{a}(z)+T_{0}\right)^{4}\right], \frac{\partial c}{\partial r}=0,(2.9) \\
z=0, r \in(0,1): u=0, v=V_{p}, \\
T=0, \frac{\partial c}{\partial z}=V_{p} \operatorname{Pe}(c-1), \\
z=\Gamma, r \in(0,1): u=0, v=V_{p}, \\
T=0, \frac{\partial c}{\partial z}=V_{p} \operatorname{Pe}\left(1-k_{0}\right) c,
\end{aligned}
$$

where $k_{0}$ is the impurity segregation coefficient and is assumed as a constant. 


\section{Numerical simulation}

A strong uniform longitudinal magnetic field can be produced approximately by four electrical coils located far from the floating full zone with $I_{0}=$ $4.0 \times 10^{4} \mathrm{~A}$ in the same direction in each coil, and the magnetic field strength in the melt zone is $0.988 \mathrm{~T}$ [6]. To design the non-uniform magnetic field, the coils are located near the free surface, each carries the equal electric current $I_{0}=2.0 \times 10^{3} \mathrm{~A}$ with the opposite direction compared with its neighbour coil, then the magnetic field vector at the middle horizontal cross-section between the two coils is purely radial. When two coils are used, it is similar to the cusped field which is used for Czochralski crystal growth (Fig. 2a). Such a configuration of the magnetic field has the character that in the melt zone, the magnetic field strength near the horizontal plane of the radial magnetic field is much stronger than the field strength in other regions.

The steady governing equations are treated as "pseudo-unsteady" equations and discretized on the staggered grids by the finite control volume difference method [7]. Fully implicit time marching is used with first order in time term. Hybrid scheme is adopted in writing the coefficients of convection and diffusion term. The "block modification" method [8] is used to solve the problem of difference equations. The boundary conditions are discretized with second order accuracy. The total grid meshes $32 \times 64$ are used according to the study elsewhere [9]. The variable convergence criteria may be estimated as follows:

$$
\begin{aligned}
& \left|\frac{u-u_{\text {old }}}{u_{\text {old }}}\right|_{\max }<1.0 \times 10^{-5},\left|\frac{v-v_{\text {old }}}{v_{\text {old }}}\right|_{\max }<1.0 \times 10^{-5}, \\
& \left|\frac{T-T_{\text {old }}}{T_{\text {old }}}\right|_{\max }<1.0 \times 10^{-5},\left|\frac{c-c_{\text {old }}}{c_{\text {old }}}\right|_{\max }<1.0 \times 10^{-5},
\end{aligned}
$$

where the subscript "old" denotes the previous iteration and the subscript "max" means the maximum value of the absolute values. The residual of each governing equation is smaller than $1.0 \times 10^{-7}$.
At first, the case of the designed magnetic field produced by two coils is studied. It brings the fundamental insights into the principle of the magnetic field design. The two coils are located, respectively, at $1.8 \leqslant z \leqslant 2.2$ and $1.2 \leqslant z \leqslant 1.6$ in the upper half zone to produce the strong radial magnetic field component there, because the convection there has a direct influence on the concentration distribution at the solidification interface. The magnetic damping effects on the flow near the free surface are investigated, and the reduction of the flow velocity on the free surface is shown in Fig. 2b. The horizontal crosssection of purely radial magnetic field is adjusted relative to the "key point", which relates to the maximum flow velocity on the free surface in the case without magnetic field. Such a design shows that in the case of two coils, the flow velocity on the free surface in the upper half zone is reduced greatly because of the strong radial magnetic field component. Moreover, the reduced flow intensity is relatively more uniform compared to either the case without magnetic field or the case of the longitudinal magnetic field. As to the lower half zone, the flow is not reduced to the same extent because of the weak magnetic damping effect there. The magnetic damping effects on the free surface in the upper half zone include two aspects: the reduction extent of the flow intensity and the uniformity of the damped flow intensity. Considering the two aspects at the same time, the case that the horizontal plane of purely radial magnetic field is located around the "key point" is chosen to represent the magnetic field produced by two coils (the solid line in Fig. 2b), in which the coils are located at $z=2.0$ and $z=1.4$. On the other hand, changing the distance between the two coils when the location of the purely radial magnetic field plane is fixed, the magnetic damping effects of the case when the two coils are located at $z=2.0$ and $z=1.4$ are also much better.

The magnetic damping effects of three types of magnetic fields on the flow velocity, temperature and concentration distributions in the melt zone are compared in Fig. 3. The concentration distributions at the solidification interface are illustrated in Fig. 4, and a non-dimensional 

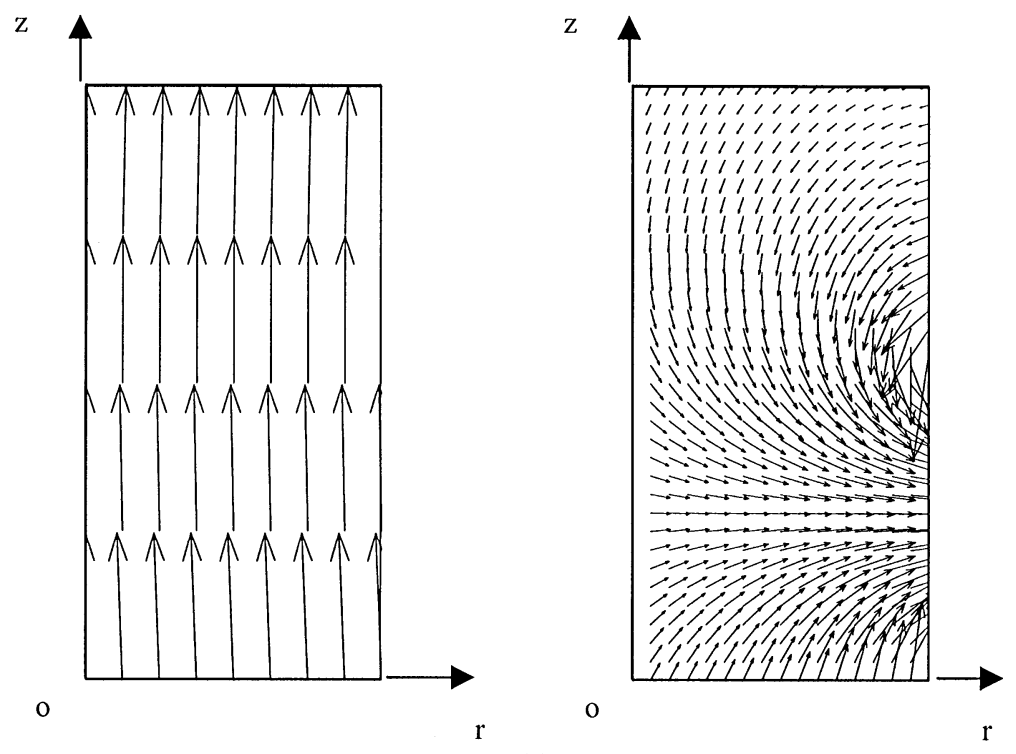

(a)

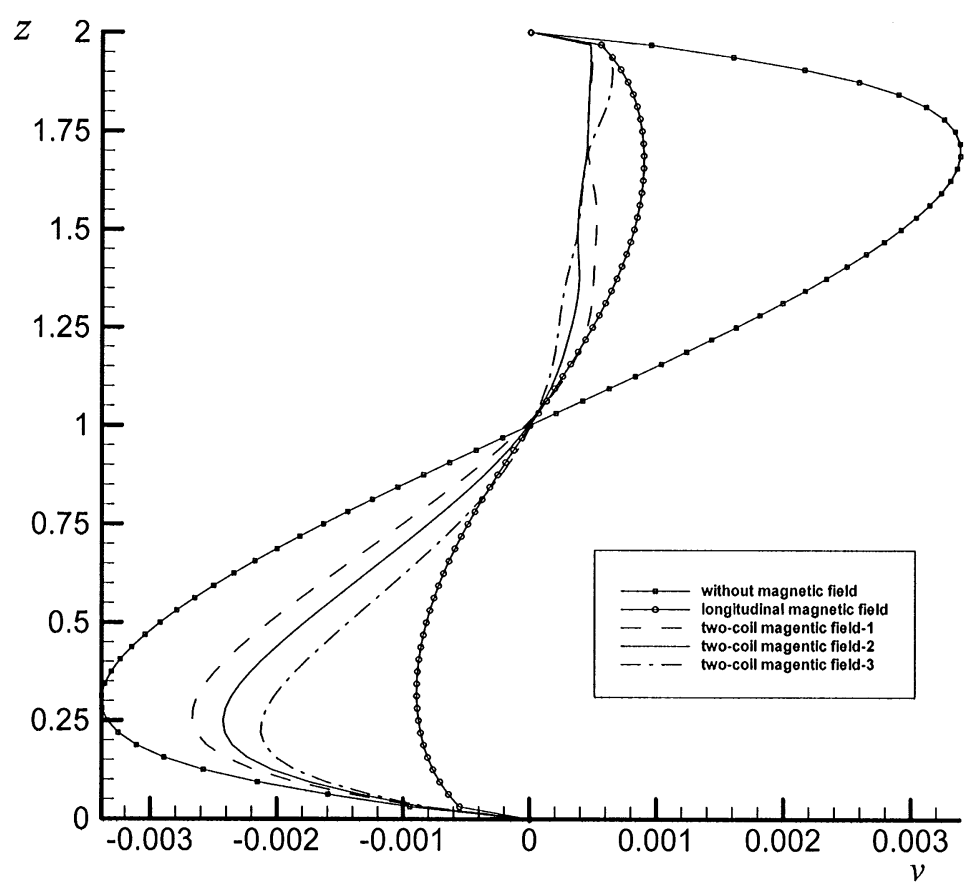

(b)

Fig. 2. Schematic diagrams of the longitudinal magnetic field and the two coil magnetic field with similar maximum field strength (a) and profiles of the flow velocity on the free surface $v$ in the cases of the two coil magnetic fields when the coils are located at: (1) $z=2.2$ and $z=1.6$; (2) $z=2.0$ and $z=1.4$; (3) $z=1.8$ and $z=1.2$, respectively. 

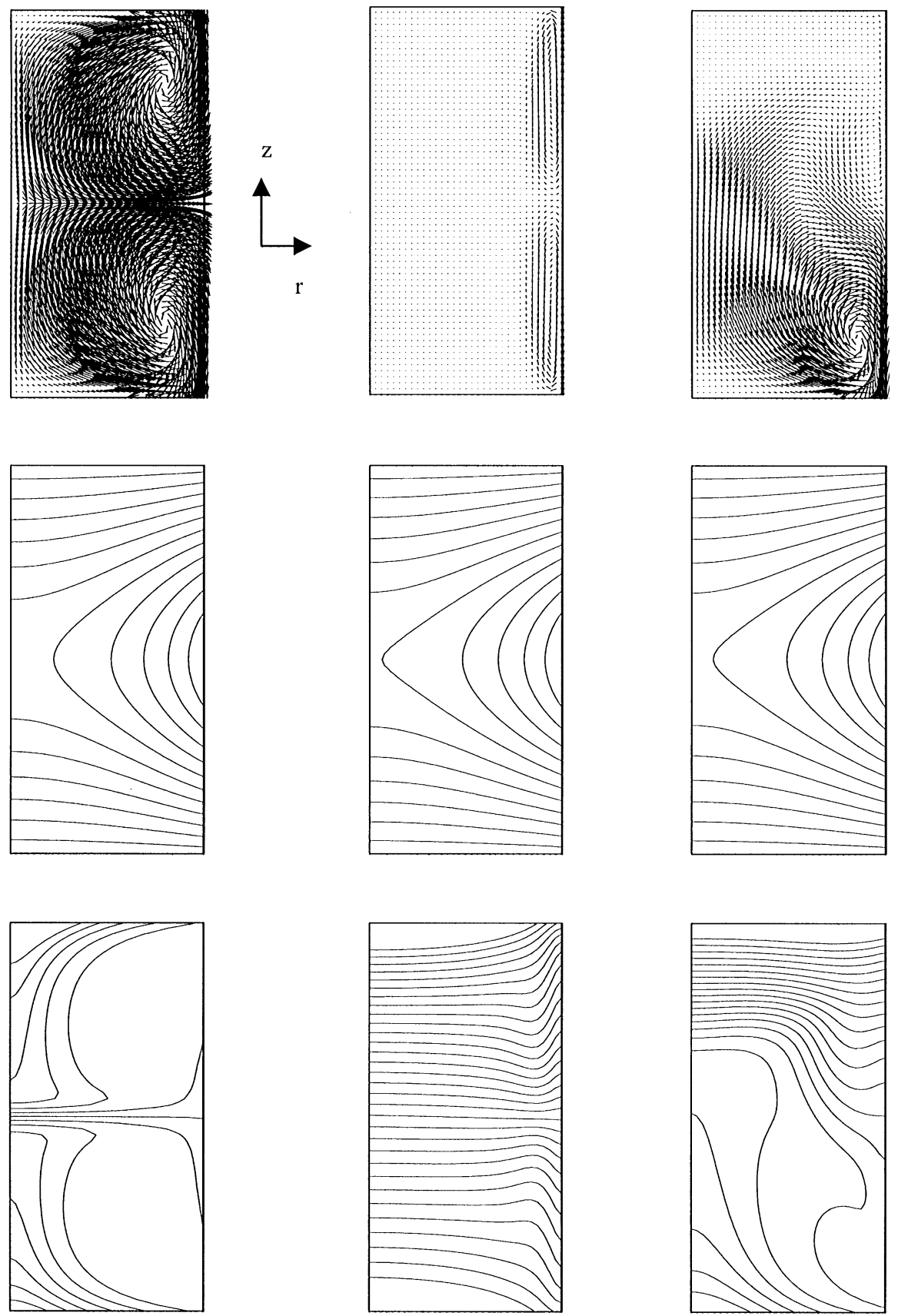

Fig. 3. Flow velocity (upper), temperature (middle) and concentration (lower) distributions in the case of the designed magnetic field produced by two coils located at $z=2.0$ and $z=1.4$ (right) compared to the cases without magnetic field (left) and the longitudinal magnetic field (middle), respectively. $\Delta T$ and $\Delta c$ in the three cases are, respectively, the same. 


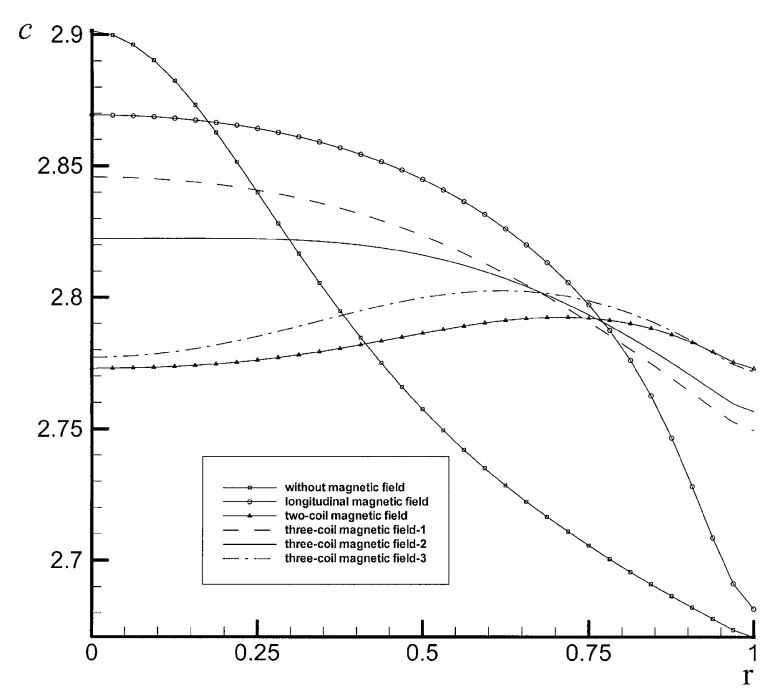

Fig. 4. Profiles of the concentration distribution at the solidification interface in the cases without magnetic field, the longitudinal magnetic field and the designed magnetic field produced by two coils located at $z=2.0$ and $z=1.4$ and the designed magnetic field produced by three coils when the coils are located at $z=2.0, z=1.4$ and $z=0.0 ; z=2.0, z=1.4$ and $z=-0.3 ; z=2.0, z=1.4$ and $z=-1.0$, respectively. The nonuniformity $\zeta$ is $8.3 \%, 6.7 \%, 0.7 \%, 3.4 \%, 2.4 \%$ and $1.1 \%$, respectively.

non-uniformity of the concentration distribution is defined as

$\zeta=\frac{c_{\max }-c_{\min }}{\bar{c}}$,

where $c_{\max }, c_{\min }$ and $\bar{c}$ are maximum, minimum and average values at the solidification interface, respectively. In the case without magnetic field, the strong convective recirculations occupy the whole melt zone, and the flow intensities along the axis and at the solidification interface are particularly non-uniform. The mass transfer under such a flow structure induces the large non-uniformity of the concentration distribution at the solidification interface. When the strong longitudinal magnetic field is applied, the flow intensity is reduced to a great extent, but the relatively strong convective recirculations still persist along the free surface. The difference between the flow intensity in the core region and the one near the free surface is so obvious that it results in the distortion of the concentration distribution near the free surface. So the non-uniformity of the concentration distribution at the solidification interface in the case of the longitudinal magnetic field is still quite large. In the case of the designed magnetic field produced by two coils, the reduced weak and uniform flow on the free surface only in the upper half zone can drive a weak upper convective recirculation. In other words, the upper convective recirculation is damped to a large extent as a whole and constrained to the area near the center of the free surface. Although the relatively strong lower convective recirculation expands its flow area into the upper half zone, it still cannot penetrate into the area in front of the solidification interface also because of the strong radial magnetic field component there. So the reduced radial flow intensity in front of the solidification interface is weak and more uniform as shown in Fig. 3, and it greatly improves the non-uniformity of the concentration distribution at the solidification interface as shown in Fig. 4.

In the case of the designed magnetic field produced by two coils, the merits of the magnetic damping effect on the concentration distribution at the solidification interface are obvious; however, the flow intensity in the lower half zone is not reduced to a sufficient extent. So, based on the designed magnetic field produced by two coils, the reduction of the lower convective recirculation is studied by the application of a third coil. When the newly produced pure radial magnetic field planes are located at the top, the middle and the bottom of the lower half zone, the effects of the designed magnetic field on the flow velocity and the concentration distribution at the solidification interface are compared to the case of the designed magnetic field produced by two coils (Figs. 4 and 5). When the distance between the two planes is small, the magnetic field in the melt zone is enhanced, especially in the lower half zone, and the whole flow velocity is reduced greatly, but the improvement of the non-uniformity in the concentration distribution at the solidification interface became worse. With an increasing distance, the smaller the enhancement of the magnetic field, the smaller the reduction extent of the lower convective recirculation, although the reduction is still obvious, and the better the improvement of 

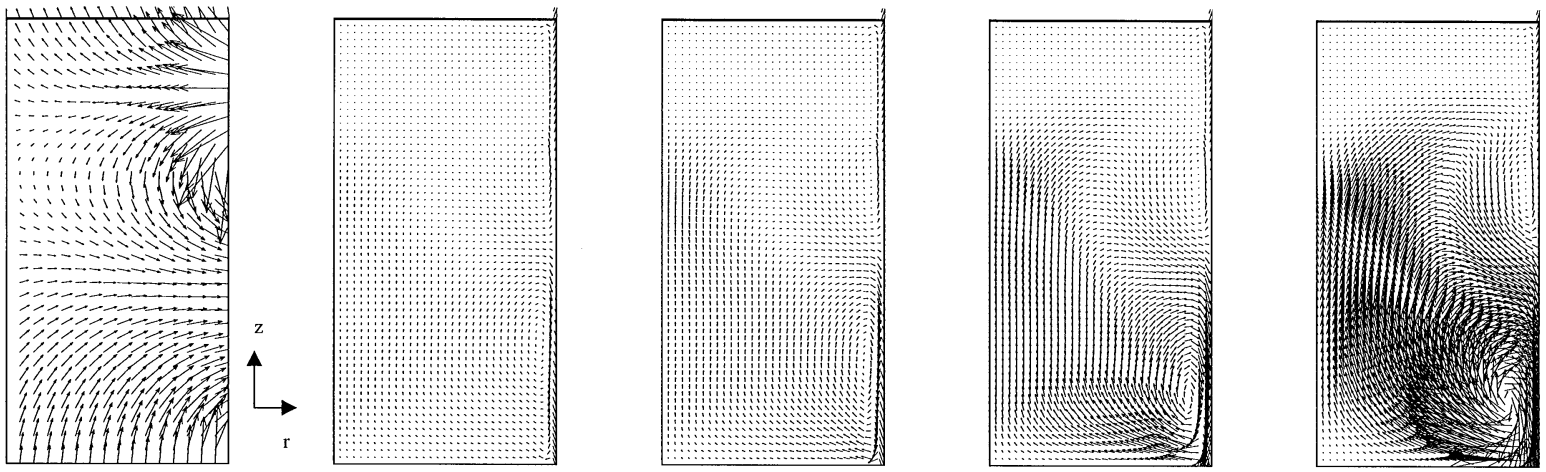

Fig. 5. Configuration of the designed magnetic field produced by three coils (a) and the flow velocity when three coils are located at $z=2.0, z=1.4$ and $z=0.0(\mathrm{~b}) ; z=2.0, z=1.4$ and $z=-0.3$ (c); $z=2.0, z=1.4$ and $z=-1.0$ (d), respectively. The flow velocity in the case of the designed magnetic field produced by two coils (e) located at $z=2.0$ and $z=1.4$ is included as reference.
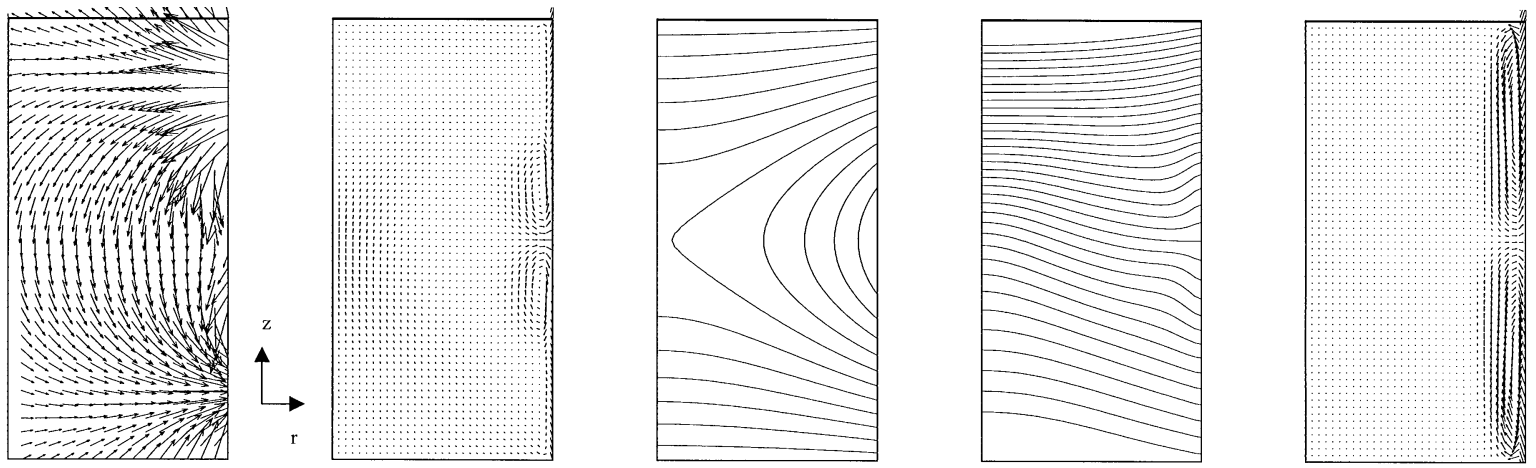

Fig. 6. Configuration of the designed magnetic field (a), the flow velocity (b), temperature (c) and concentration distributions (d) by four coils located, respectively, at $z=2.0, z=1.4, z=0.6$ and $z=0.0$. The flow velocity in the case of the longitudinal magnetic field (e) is included as reference.

the non-uniformity in the concentration distribution at the solidification interface. Seen from the comparison above, it seems that the flow in the melt zone which remains with some intensity can result in the better mixture of the concentration distribution in the body of the melt zone. So there must be a compromise between the reduction of the flow velocity and the improvement of the concentration distribution at the solidification interface, for example, the solid line in Fig. 4. Following such considerations, a magnetic field produced by four coils is designed for the floating full zone crystal growth. Based on the designed magnetic field produced by two coils, the second pair of coils is also located symmetric to the corresponding "key point" in the lower half zone. Its effects on reducing the flow velocity, temperature and concentration distributions are shown in Fig. 6, and the concentration distributions at the solidification interface are illustrated in Fig. 7. In the case of the designed magnetic field produced by four coils, the flow velocity is greatly reduced to a comparable extent through the locally direct reduction of the flow near the free surface compared with the case of the longitudinal magnetic field. The reduced weak convective recirculations are constrained in the area around the center of the free surface and leave the weak flow in front of the solidification interface with relatively uniform intensity. The much more 


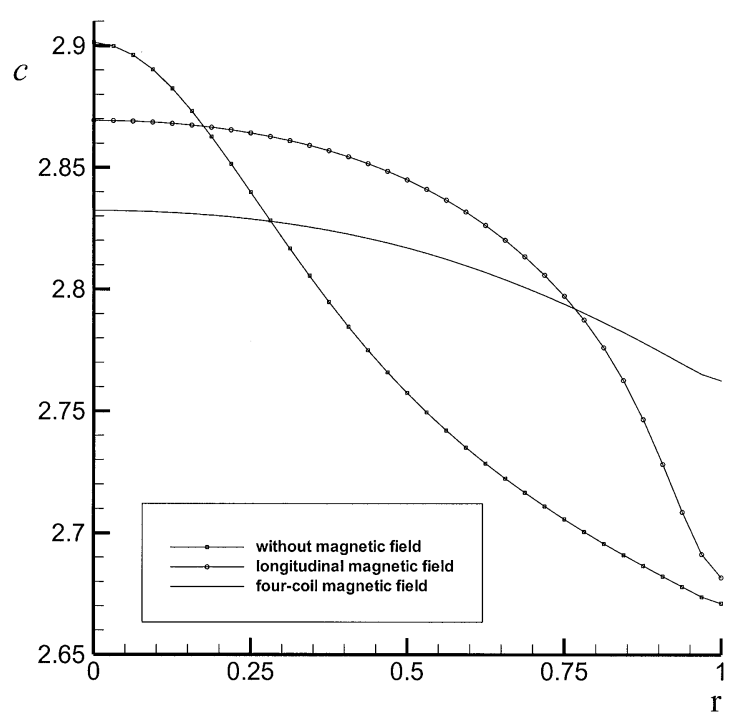

Fig. 7. Profiles of the concentration distribution at the solidification interface in the cases without magnetic field, the longitudinal magnetic field and the designed magnetic field produced by four coils when the coils are located at $z=2.0$, $z=1.4, z=0.6$ and $z=0.0$, respectively. The non-uniformity $\zeta$ is $8.3 \%, 6.7 \%$ and $2.5 \%$, respectively.

reasonable flow structure compared with the case of the longitudinal magnetic field results in the great improvement of the uniformity in the concentration distribution at the solidification interface as shown in Fig. 7. Compared to the case of the magnetic field produced by two coils, the improvement of the concentration uniformity is a bit worse, it may be due to the relatively poor mixture of the impurity by the convection in the body of the melt zone. However, taking into account the large reduction extent of the flow intensity in the melt zone, the case of the magnetic field produced by four coils has attained a good compromise between the two aspects of the magnetic damping effects.

\section{Discussion}

The main idea of the present paper is to locate the coil as close to the free surface as possible to generate the inhomogeneous magnetic fields which are specially designed to reduce the flow near the free surface, and then in the floating zone. The results of the numerical simulation show that with the approach of the magnetic field design, it is expected to improve the radial macro-segregation and reduce convection in the melt at the same time which are better than those of the case of a uniform longitudinal magnetic field. On the other hand, from the viewpoint of practice, the high temperature melt of the floating zone prevents the coils from being placed too closely to the free surface of the melt. A careful design should be applied if the idea of magnetic design can be used in practice. A space experiment of semiconductor crystal growth has been performed for a rod of $50 \mathrm{~mm}$ in diameter [10]. As an example, we adopted the radius of semiconductor and then the free surface location at $R_{0}=4 \mathrm{~cm}$, and the radius of the electrical coils located at $1.5 R_{0}=$ $6 \mathrm{~cm}$ for producing the designed magnetic field as discussed in the present paper. In this case, there is enough room to arrange the coils with enough current such as $I_{0}=10^{4} \mathrm{~A}$.

The floating zone method is beneficial to a high quality semiconductor production on the ground, but the gravity limits the diameter of the sample and the buoyancy convection may induce the concentration distribution non-uniformity in the sample. The microgravity environment greatly reduces buoyancy convection, and the thermocapillary convection may also be oscillatory in the case of a larger temperature difference or the Marangoni number. Therefore, introducing a magnetic field to improve the velocity and concentration distributions of the sample is important even in microgravity environment. The conclusion of the present paper shows that, the effect of a designed magnetic field will be better than that of the longitudinal magnetic field, which is usually adopted. It should be noted that a simplified model to suggest the idea of a designed magnetic field is discussed in the present paper, and more analyses to improve the simplified model to relate the practice are necessary, and will be discussed elsewhere. Moreover, further study about the influence of the solidification interface shape on the magnetic damping effects on the velocity, temperature and impurity distributions is also necessary. 


\section{Acknowledgements}

The research is supported by the project 95-yu34 of the Ministry of Science and Technology of China and grant (19789201) of the National Natural Science Foundation of China.

\section{Appendix}

Physical properties of $\mathrm{P}$-doped $\mathrm{Si}$

$$
\begin{aligned}
& T_{0}=1683 \mathrm{~K} \\
& \Delta T=10 \mathrm{~K} \\
& \rho=2.53 \mathrm{~g} / \mathrm{cm}^{3} \\
& v=0.0035 \mathrm{~cm}^{2} / \mathrm{s} \\
& \kappa=0.255 \mathrm{~cm}^{2} / \mathrm{s} \\
& k=0.67 \mathrm{~W} / \mathrm{cm} \mathrm{K} \\
& \sigma_{T}=-1.3 \times 10^{-4} \mathrm{~N} / \mathrm{m} \mathrm{K} \\
& D=5.0 \times 10^{-8} \mathrm{~cm}^{2} / \mathrm{s} \\
& k_{0}=0.35 \\
& \varepsilon=0.3 \\
& \sigma_{\mathrm{e}}=1.23 \times 10^{6} \Omega^{-1} \mathrm{~m}^{-1} \\
& V_{p}=5.0 \times 10^{-6} \mathrm{~m} / \mathrm{s}
\end{aligned}
$$

\section{References}

[1] N. de Leon, J. Guldberg, J. Salling, J. Crystal Growth 55 (1981) 406.

[2] D.G. Robertson Jr., D.J. OConnor, J. Crystal Growth 76 (1986) 111.

[3] A. Croll, P. Dold, K.W. Benz, J. Crystal Growth 137 (1994) 95.

[4] K. Li, W.R. Hu, J. Crystal Growth 222 (2001) 677.

[5] R.W. Series, D.T.J. Hurle, J. Crystal Growth 113 (1991) 305.

[6] C.W. Lan, J. Crystal Growth 169 (1996) 269.

[7] D.B. Spalding, Int. J. Numer. Meth. Eng. 4 (1972) 551.

[8] S.V. Patankar, Advanced Computational Heat Transfer and Fluid Flow, Graduate Student Course 8352, Mechanical Engineering Department, University of Minnesota, USA.

[9] K. Li, W.R. Hu, Effect of non-uniform magnetic field on crystal growth by floating zone method in microgravity, Science in China, A31 (2001) 466.

[10] E.V. Markov, V.Yu. Antropov, V.M. Biryukov, V.A. Goncharov, Yu.N. Dyakov, V.A. Pilgoon, V.A. Pesikov, V.P. Chegnov, A.I. Ivanov, V.P. Nikitsky, Proceedings of the Joint X European and VI Russian Symposium on Physical Sciences in Microgravity, St. Petersburg, Russia, Vol. 2, 1997, p. 11. 